\title{
Effect of a Strong Axial Magnetic Field in the Plasma Recombination and Extreme Ultraviolet Emission from a Highly-Ionized Capillary Discharge
}

MARIO C. MARCONI, JORGE J. ROCCA, MEMBER, IEEE, JOHN F. SCHMERGE, MAYO VILLAGRAN, AND FRANK J. LEHMANN

\begin{abstract}
The effect of an externally-applied 70-100 kG axial magnetic field in the temporal evolution of the extreme ultraviolet emission from a $500 \mu \mathrm{m}$ diameter highly-ionized LiH capillary discharge has been studied. In the absence of external magnetic confinement, strong emission from ionic transitions excited by collisional recombination is observed at the end of the current pulse. The externally-applied magnetic field is observed to reduce the intensity of the recombination lines by decreasing the rate of plasma cooling by electron heat conduction to the capillary walls. In contrast, the self-generated magnetic field of the discharge aids to the generation of an initially hot plasma, and allows rapid conduction cooling at the end of the current pulse. The results are discussed in relation to a proposed capillary-dischargeexcited extreme ultraviolet recombination laser scheme.
\end{abstract}

\section{INTRODUCTION}

$\mathrm{L}$ ASER radiation at soft $X$-ray wavelengths has been demonstrated in highly-ionized plasmas generated by high-energy laser pulses. Both collisional electron excitation [1]-[3] and collisional electron-ion recombination schemes [4]-[7] have been successfully employed to create population inversions. These experiments used larger laser systems as the pumping source, in some cases the same lasers used for fusion research.

Fast capillary discharges can efficiently produce dense, highly-ionized plasmas with a large length-to-diameter ratio [8]-[13] and are of interest to explore the possibility of generating extreme ultraviolet (XUV) laser radiation in a compact discharge-excited device [14]. In a capillary discharge geometry, hot plasmas created during high-current density pulses are a potential medium for the generation of population inversions by collisional electron excitation, while rapid cooling of the plasma column at the

Manuscript received May 7, 1990. This work was supported by the $\mathrm{Na}$ tional Science Foundation under Grant ECS-8606226 and the National Science Foundation under a Presidential Young Investigator Award granted to J. J. Rocca, M. C. Marconi was supported by the Universidad de Buenos Aires and the Consejo Nacional de Investigaciones Cientificas y Tecnicas de la Republica Argentina.

M. C. Marconi, J. J. Rocca, J. F. Schmerge, and M. Villagran are with the Department of Electrical Engineering, Colorado State University, Fort Collins, CO 80523

F. J. Lehmann was with the Department of Electrical Engineering, Colorado State University, Fort Collins, CO 80523 . He is now with the Lock heed Advanced Development Company, Burbank, CA 91520.

IEEE Log Number 9038282. end of the excitation could lead to population inversion by collisional recombination.

The collisional recombination laser excitation scheme [15] is particularly attractive for achieving an efficient utilization of the pumping energy because it can transfer a significant fraction of the energy stored in ground-state ions into the upper-laser level [16]. Ground-state ions can be efficiently generated in a plasma and when the plasma cools collisional recombination of these ions with plasma electrons predominantly populates the higher energy levels, originating population inversions. Due to the strong dependence of the collisional recombination rate on the inverse of the electron temperature [17], rapid cooling of the plasma is a key requirement for the generation of large inversions. In the case of plasmas generated by laser ablation, three different cooling mechanisms have been reported to be successfully used in generating amplification at soft $\mathrm{X}$-ray wavelengths by collisional recombination: adiabatic expansion of the plasma [5], electron heat conduction [7], and radiation losses [1]. The highest energy soft X-ray laser pulse reported corresponds to the $18.2 \mathrm{~nm}$ line of CVI in a magnetically-confined radiatively-cooled plasma column [1]. The externally-applied magnetic field was used to reduce charged particle diffusion, thereby maintaining the density of the laser created plasma relatively constant, as inelastic collisions followed by radiative decay cooled the electron gas.

In recent experiments with highly-ionized capillary-discharge plasmas [10], we have observed the emission from XUV radiative transitions excited by collisional recombination at the end of the current pulse. Time-resolved XUV spectroscopy of a $500 \mu \mathrm{m}$ diameter LiH capillary plasma excited by a 45 ns FWHM 5 J current pulse showed OVI impurity lines to be dominant in the spectra at times near the peak of the current pulse, while lines from lesser ionized species excited by collisional recombination were the most intense during the decay of the current pulse [7]. Herein we discuss the effect of an intense ( 70-100 kG) externally-applied axial magnetic field on the XUV emission associated with plasma recombination in a $500 \mu \mathrm{m}$ diameter $\mathrm{LiH}$ capillary discharge. This is, to our knowledge, the first study of the evolution of a capillary-discharge plasma confined by a strong axial 
magnetic field. The results of the experiments discussed in this paper demonstrate the dominant role of electron heat conduction in cooling the capillary discharge plasma.

The capillary discharge setup employed in this experiment was similar to that we used in previous studies which did not include an externally-applied magnetic field [12], [13]. The plasma was generated in a lithium hydride capillary $76 \mathrm{~mm}$ long and $500 \mu \mathrm{m}$ in diameter, excited by a $5 \mathrm{nF}$ ring of ceramic capacitors. The capacitors were connected to two hollow graphite electrodes placed at the end of the capillary through a compact triggered spark-gap switch pressurized with $\mathrm{SF}_{6}$, which was not part of the setup in the previous experiments. While this switch causes an undesirable increase in the discharge circuit inductance relative to our previous experiments, thereby increasing the discharge current pulsewidth, it also makes the discharge firing very reliable, allowing an effective synchronization of the discharge pulse with the peak of the magnetic field. The inclusion of the pressurized spark gap in the discharge circuit also allowed us to perform discharges applying voltage values above the self breakdown voltage of the capillary.

An intense axial magnetic field of up to $106 \mathrm{kG}$ was generated by discharging a $400 \mu \mathrm{F}$ capacitor through a compact solenoid $10 \mathrm{~cm}$ in length that closely surrounds the capillary, as illustrated in Fig. 1. The magnetic field discharge circuit was controlled by a sealed pressurized triggered spark-gap and had a current half cycle of 260 $\mu \mathrm{s}$, thereby providing a practically constant magnetic field during the much shorter time of the capillary discharge. When the capacitor was charged to $4 \mathrm{kV}$, the peak current circulating through the solenoid reached $16 \mathrm{kA}$, corresponding to a magnetic field intensity of $106 \mathrm{kG}$. The magnetic field was measured using a precalibrated Hall probe located in the place normally occupied by the discharge channel. The magnetic field values measured with the Hall probe was found to be in good agreement with those calculated based on the value of the current that circulates through the solenoid.

The synchronization of the capillary discharge current pulse and the peak intensity of the magnetic field was achieved by first firing the spark gap in the magnetic field circuit and then, after a time interval set by a delay generator, the capillary discharge. The timing of the capillary discharge current pulse relative to the peak of the magnetic field was monitored by recording the addition of the signals from a capacitive probe that monitored the voltage of the capillary discharge capacitors and the signal from the Rogowski coil that monitored the current through the solenoid. Fig. 2(a) shows the first half cycle of the current pulse through the solenoid. A sudden step down in the sinusoidal signal of the coil current indicates the moment when the voltage of the capacitors drops due to the capillary discharge. This signal also indicates the value of the current through the solenoid and consequently the value of the external magnetic field during the discharge.

The capillary discharge volume was pumped from both ends with a turbomolecular pump to pressures in the range of $10^{-5}$ torr and was connected through a vacuum duct to

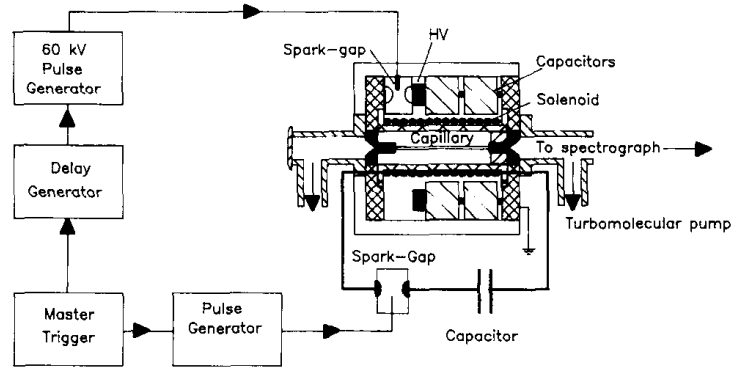

Fig. 1. Schematic representation of the experimental setup. The delay generator synchronizes the capillary discharge firing with the maximum of the magnetic field pulse.

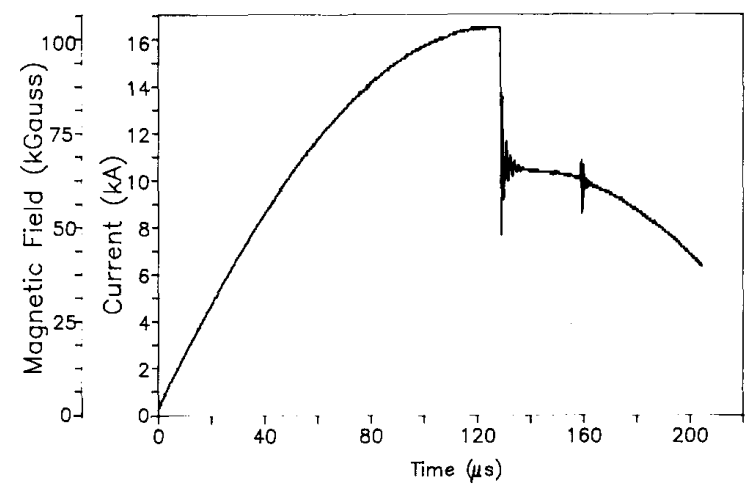

(a)

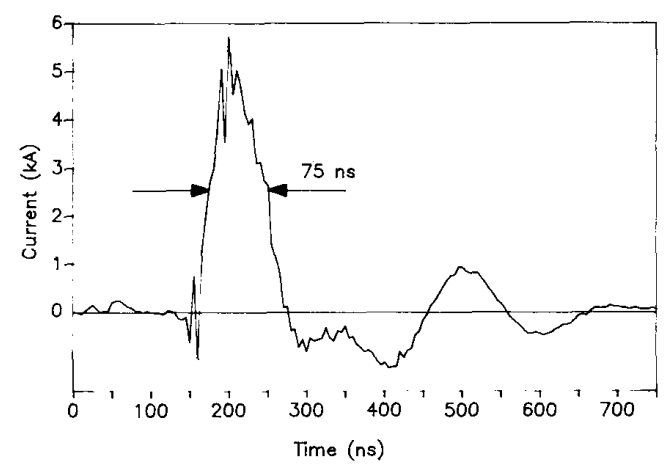

(b)

Fig. 2. (a) Oscillograph showing the signal resulting from the addition of the current pulse through the solenoid and the voltage of the capillary discharge capacitors. The sudden step down indicates the firing of the capillary discharge occurring at the peak of the magnetic field. The axis of the right can be used to determine the value of the magnetic field circuit current and the magnetic field intensity at the time of firing of the capillary discharge. (b) Capillary discharge current pulse. The discharge voltage is $55 \mathrm{kV}, B=0$.

a $1 \mathrm{~m}$ focal length normal incidence vacuum spectrograph placed inside a Faraday room along with all the other diagnostic and data gathering apparatus. Efficient light gathering was obtained using an aluminum-coated spherical mirror at grazing incidence to focus the axially emitted capillary radiation in the slit of the spectrograph. The mirror also protected the spectrograph grating from the debris produced by the discharge [18]. Time-resolved 
spectra with approximately $5 \mathrm{~ns}$ time resolution were obtained by gating a windowless dual multichannel plate intensified array detector [19]. The time evolution of the intensity of selected spectral lines was also monitored using a vacuum UV photomultiplier furnished with a sodium salicylate coated window. The gating pulse to the multichannel intensifier and the signal from a Rogowski coil that monitors the capillary current pulse were recorded and stored for every discharge shot using a 200 Msample/s dual-channel transient digitizer, allowing the determination of the recording time of the spectra relative to the current pulse. A typical capillary discharge pulse for a $55 \mathrm{kV}$ charging voltage is illustrated in Fig. 2(b), having a peak of $4.9 \mathrm{kA}$ and a full width at half maximum of $75 \mathrm{~ns}$. The peak of the current pulse occurs at $42 \mathrm{~ns}$ and the current is zero at $114 \mathrm{~ns}$ from the initiation of the pulse.

We observed, as it was previously reported for a similar discharge [12], that OVI 103.2 and $103.7 \mathrm{~nm}$ impurity lines dominate the 52-110 nm spectral region at the time of the peak of the current pulse. Using a simple collisional-radiative model and taking into account the rise time of the discharge pulse, it is possible to show that the axial region of the capillary plasma must reach a temperature of at least $27 \mathrm{eV}$ to produce a spectrum dominated by OVI lines.

The discharge generated in the presence of a strong axial magnetic field were observed to have prolonged emission from the lines associated with the highest degree of ionization observed during the discharge (OVI). Fig. 3 illustrates the temporal evolution of the intensity of the OVI $103.2 \mathrm{~nm}$ line corresponding to discharges with $B=$ $100 \mathrm{kG}, B=70 \mathrm{kG}$, and $B=0$ axial magnetic fields measured using the photomultiplier. A significant increase in the FWHM of the OVI light signal is observed when the external magnetic field is applied, almost doubling its persistence for $100 \mathrm{kG}$ compared with the $B=$ 0 case. The prolonged emission is a consequence of the reduction of charged particle diffusion to the walls due to magnetic confinement and also, as shown below, of reduced plasma cooling.

The temporal evolution of the XUV emission in the wavelength range from 68 to $110 \mathrm{~nm}$ of the $\mathrm{LiH}$ capillary for a magnetically confined discharge $(B=70 \mathrm{kG})$ and for a discharge without an externally applied magnetic field $(B=0)$ is shown in Fig. 4. These spectra were obtained using a 600 lines $/ \mathrm{mm}$ grating, providing a spectral resolution of $0.04 \mathrm{~nm} /$ channel in the diode array detector. The time delay of each spectrum relative to the beginning of the current pulse are indicated with an uncertainty of $5 \mathrm{~ns}$ set by the resolution of the transient digitizer used to record the signals. At $48 \mathrm{~ns}$ time delay, corresponding approximately to the peak of the current pulse, there is no significant difference between the $B=0$ and the $B=70 \mathrm{kG}$ spectra. The OVI 103.2 and $103.7 \mathrm{~nm}$ lines dominate the spectrum in both cases. The similarity of the XUV spectra at the time near the current peak is not surprising, since even with no externally applied magnetic field, the plasma is still being magnetically confined

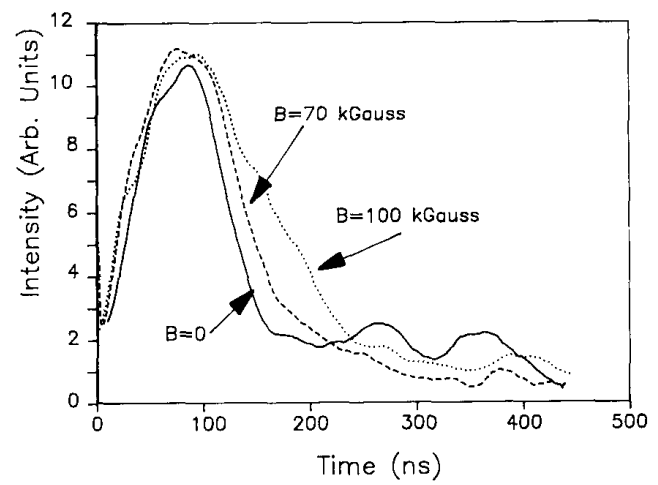

Fig. 3. Temporal evolution of the $103.2 \mathrm{~nm}$ OVI line intensity with $B=0, B=70 \mathrm{kG}$ and $B=100 \mathrm{kG}$ externally applied axial magnetic field.

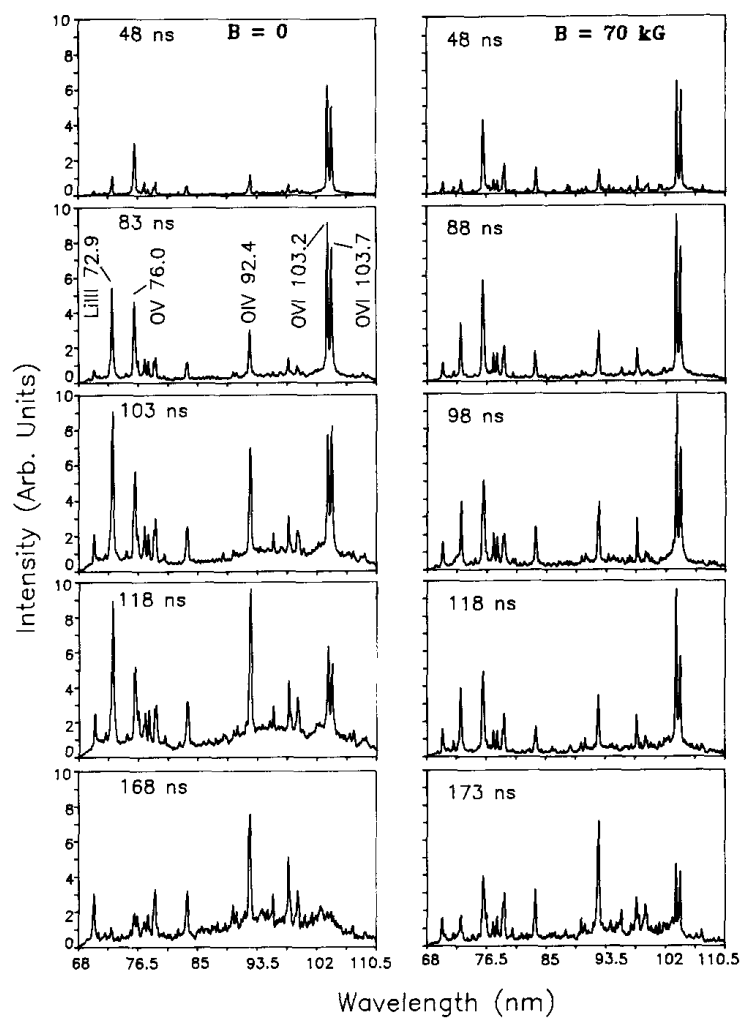

Fig. 4. Temporal evolution of the capillary discharge extreme ultraviolet emission for an externally applied magnetic field $B=0$ and $B=70 \mathrm{kG}$.

The times delay indicated in each spectrum is relative to the beginning of the current pulse. The current has its peak at $42 \mathrm{~ns}$ and is zero at 114 ns.

by the self-generated field of the capillary discharge current, which, for a $5 \mathrm{kA}$ discharge through a $500 \mu \mathrm{m} \mathrm{di-}$ ameter capillary, reaches $40 \mathrm{kG}$.

Nevertheless, Fig. 4 shows that at later times, as the discharge current decreases, the influence of the externally applied magnetic field becomes significant in controlling the plasma evolution. At $118 \mathrm{~ns}$ after the initiation 


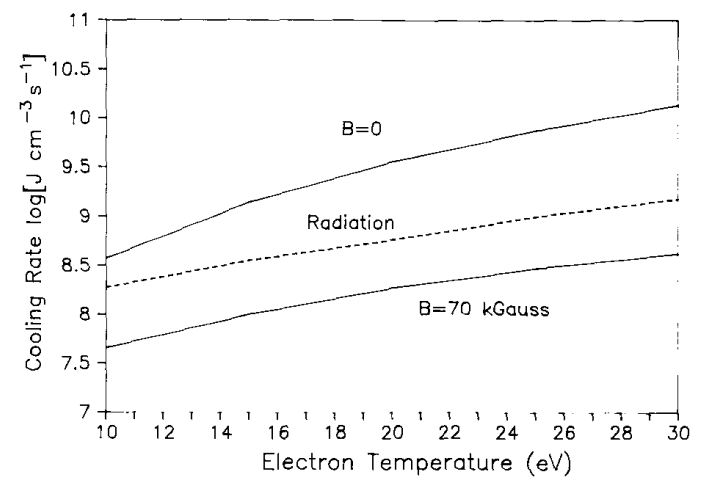

Fig. 5. Comparison of the calculated plasma cooling rate by electron heat conduction (solid lines) and radiation (dashed lines) for a discharge with $B=0$ and with a $B=70 \mathrm{kG}$ axial magnetic field. The capillary diameter is $500 \mu \mathrm{m}$ and the density of OVI, LiIII and totally ionized $\mathrm{Li}$ atoms are assumed to be equal to $1 \cdot 10^{17} \mathrm{~cm}^{-3}$.

of the current pulse, shortly after the time when the current is zero, the spectrum corresponding to $B=0$ shows strong excitation of the transition at $72.9 \mathrm{~nm}$ and also intense emission in the neighborhood of $92.3 \mathrm{~nm}$. A more detailed observation of the $92.3 \mathrm{~nm}$ emission utilizing a 2400 lines/mm grating for higher spectral resolution, allowed us to identify it with OIV transitions at 92.13 and $92.34 \mathrm{~nm}$. The emission at $72.9 \mathrm{~nm}$ corresponds to the 3-2 transition of LillI with a possible contribution from the OV line at $72.89 \mathrm{~nm}$ which cannot be resolved with the spectrograph used in this experiment. Both the 72.9 $\mathrm{nm}$ line and the OIV $92.3 \mathrm{~nm}$ emission are nearly one order of magnitude more intense at the end of the current pulse than at the peak of the current pulse. This behavior is consistent with excitation following collisional electron-ion recombination as the plasma cools. In contrast, the spectra taken with approximately the same time delay when a $70 \mathrm{kG}$ axial magnetic field was applied show nowhere near the same increase in the intensity of the recombination excited lines. Moreover, the spectra corresponding to the $B=0$ discharge present a recombination continuum of significant intensity which is considerably weaker in the case of $B=70 \mathrm{kG}$ spectra. Data obtained in the presence of a $100 \mathrm{kG}$ magnetic field display similar effects.

Computation of the plasma cooling rates show that the reduced recombination observed in the magnetically confined discharges is a consequence of a large reduction in the plasma electron heat conduction cooling rate, as discussed below. Fig. 5 illustrates the calculated magnitude of the electron heat conduction and radiative plasma cooling rates as a function of the electron temperature for a capillary plasma $500 \mu \mathrm{m}$ in diameter having a plasma density of $1 \cdot 10^{18} \mathrm{~cm}^{-3}$. The electron heat conduction rates were computed using Braginskii's heat conduction coefficients [20]. The radiative cooling rate due to the hydrogenic lithium ions was calculated using a collisional-radiative model which includes all excited states having principal quantum numbers $n$ up to 12 , while the radiative power loss coefficient calculated by Keane and Skinner [21] was used to approximate the electron cooling rate due to oxygen ions.

The cooling rates were estimated for the case in which the densities of OIV, LiIII, and totally ionized Li ions are equal to $1 \cdot 10^{17} \mathrm{~cm}^{-3}$, since these densities are likely to be a fair representation of the conditions existing shortly after the peak of the discharge current peak. Fig. 5 shows that in the absence of a strong magnetic field, electron heat conduction to the capillary walls is the dominant plasma cooling mechanism. The $70 \mathrm{kG}$ axial magnetic field causes an important reduction of the electron heat conduction rate, resulting in reduced plasma recombination which is in agreement with the spectra presented in Fig. 4. Computations done for other ionic distributions that might possibly exist in the discharge, such as more highly ionized plasmas in which the ratio of Li III to totally ionized $\mathrm{Li}$ is $1: 4$, also show a similar decrease in the plasma cooling rate when the axial magnetic field is applied. While in Fig. 5 the cooling rates for all plasma temperatures were calculated assuming a constant degree of ionization and constant ratio of the population of the different ions, during the evolution of the capillary plasma these ratios will change. That is, as the plasma cools the actual degree of ionization decreases, and consequently, at lower temperatures the radiative cooling rate in the discharge can be expected to be lower than the value in Fig. 5 . In any case, the important reduction in the plasma cooling rate caused by the $70 \mathrm{kG}$ applied magnetic field is clearly illustrated by the simple calculations described above.

In a small diameter capillary discharge geometry where the plasma is closely surrounded by the walls, electron heat conduction is frequently the dominant plasma cooling mechanism. In this situation, a strong externally applied axial magnetic field diminishes the electron heat transport coefficient causing an important reduction in the plasma cooling rate which consequently decreases the plasma recombination rate at the end of the discharge cur- 
rent pulse. Consequently, in a capillary discharge recombination laser scheme in which conduction cooling is the dominant power loss mechanism, an externally applied magnetic field would have a negative effect in the generation of population inversions. The opposite effect can be expected in the case of a capillary discharge in which radiation losses is the dominant plasma cooling mechanism. In this case, a strong externally applied axial magnetic field is likely to enhance the creation of population inversions by reducing charged particle diffusion and maintaining a high density as the plasma cools, as has already been reported for a magnetically confined laser created plasma column [4].

While the externally applied magnetic field is not beneficial in a conductively-cooled capillary plasma recombination laser scheme, the self-generated magnetic field generated by the capillary discharge current is ideally suited. That is, the magnitude of the electron heat conduction is minimized during the current pulse by the selfgenerated magnetic field, thereby allowing for the generation of an initially hot plasma. Also, at the end of the current pulse, when rapid plasma cooling is required, the intensity of the self-generated magnetic field decreases and the electron heat conduction increases significantly, resulting in rapid cooling of the plasma and intense emission from transitions excited by collisional recombination. Under adequate plasma conditions, the rapid plasma cooling by electron heat conduction, which is responsible for the intense collisional recombination induced emission observed in the $B=0$ case experiments, could result in XUV amplification.

\section{ACKNOWLEDGMENT}

The authors want to thank G. Skinner for his collaboration in the design of the solenoid used in the generation of the magnetic field and D. Beethe for his assistance in the calculation of the cooling rates.

\section{REFERENCES}

[1] D. L. Matthews, P. L. Hagelstein, M. D. Rosen, M. J. Eckart, N. M. Ceglio, A. V. Hazi, H. Medecki, B. J. MacGowan, J. E. Trebes, B. L. Whitten, E. M. Campbell, C. W. Hatcher, A. M. Hawryluk, R. L. Kauffman, L. D. Pleasance, G. Rambach, J. H. Scofield, G. Stone, and T. A. Weaver. "Demonstration of soft X-ray Amplifiers," Phys. Rev. Lett., vol. 54, pp. 110-113, 1985.

[2] T. N. Lee, E. A. McLean, and R. L. Elton. "Soft X-ray lasing in neonilike germanium and cooper plasmas," Phys. Rev. Lett., vol. 59, pp. 1185-1188, 1987.

[3] B. J. MacGowen, S. Maxon, P. L. Hagelstein, C. J. Keane, R. London, D. L. Matthews, M. D. Rosen, J. H. Scofield, and D. A. Whelan. "Demonstration of soft X-ray amplification in nickel-like Ions," Phys. Rev. Lett., vol. 59, pp. 2157-2160, 1987.

[4] S. Suckewer, C. H. Skinner, H. Milchberg, C. Keane, and D. Voorhes, "Amplification of stimulated soft X-ray emission in a capillary plasma column,"' Phys. Rev. Lett., vol. 55, pp. 1753-1756, 1985.

[5] C. Chenais-Popovics, R. Corbett, C. J. Hooker, M. H. Key, G. P. Kiehm, C. L. S. Lewis, G. J. Pert, C. Regan, S. Sadaat, T. Tomie, and D. Willi. "Laser amplification at $18.2 \mathrm{~nm}$ in recombining plasma from a laser-irradiated carbon fiber," Phys. Rev. Lett., vol. 59, pp. 2161-2164, 1987.

[6] P. Jaeglé, G. Jamelot, A. Carillon, A. Klisnick, A. Sureau, and H. Guennov, "Soft X-ray amplification by lithium-like ions in recom- bining hot plasmas," J. Opt. Soc. Amer. B, vol. 4, pp. 563-574, 1987.

[7] H. Milchberg, C. H. Skinner, S. Suckewer, and D. Voorhees, "Measurements of population inversion and gain in carbon fiber plasmas," Appl. Phys. Lett., vol. 47, p. 1151, 1985

[8] H. Conrads, "Die erzeugung von seriengrenzkontinua mit hilfe von gleitfunken zur absolut messung in vakuum-ultraviolett,"' Zeitschrift fur Physik, vol. 200, pp. 444-445, 1967.

[9] P. Bogen, H. Conrads, G. Gatti, and W. Gatti, and W. Kohlhass, "Continuum radiation source of high intensity," J. Opt. Soc. Amer., vol. 58, pp. 203-206, 1968.

[10] R. A. McCorkle, "High-Power sliding spark capillary discharge in vacuum: Variations and applications," Appl. Phys. A, vol. 26, pp. $261-270,1981$

[11] S. M. Zakharov, A. A. Kolomenskii, and A. I. Samoklin, "Soft X-ray emission in a high-current capillary discharge,"'Sov. Technol. Phys. Lett., vol. 6, pp. 486-487, 1980.

[12] M. Marconi and J. J. Rocca. "Time resolved extreme ultraviolet emission from a highly ionized lithium capillary discharge," Appl. Phys. Lett., vol. 54, pp. 2180-2182, 1989.

[13] J. J. Rocca, M. C. Marconi, M. Villagran Muniz, and D. C. Beethe, "Capillary discharge plasmas as extreme ultraviolet laser sources," OSA Proceedings on Short Wavelength Coherent Radiation: Generation and Applications, R. Falcone and J. Kirz, Eds., 1988, pp. 99105.

[14] J. J. Rocca, D. C. Beethe, and M. C. Marconi, "Proposal for soft X-ray and XUV lasers in capillary discharges," Opt. Lett., vol. 13, pp. 565-567, 1988.

[15] L. I. Gudzenko and 1. A. Shepelin, "Negative absorption in nonequilibrium hydrogen plasma," Zh. Eksp. Teor. Fiz., vol. 45, pp. 1445-1449, 1963, (Sov. Phys. JEPT, vol. 18, p. 998, 1964).

[16] W. T. Silfvast and O. R. Wood II. "Recombination lasers in the vacuum ultraviolet," in Laser Techniques for Spectroscopy, AIP Conf. Proc., T. J. Mcllrath and R. R. Freeman, Eds., 1982.

[17] H. R. Griem, Ed. Plasma Spectroscopy. New York: McGraw, 1964

[18] M. C. Marconi, J. F. Schmerge, and J. J. Rocca, "Light collection and wavelength calibration for an extreme ultraviolet spectrograph," Rev. Scientif. Instrum., vol. 60, pp. 966-969, 1989.

[19] M. C. Marconi, J. J. Rocca, and G. J. Krause, "Fast gating of a windowless dual-multichannel-plate-intensified array detector,' $J$. Phys. E: Scientif. Instrum., vol. 22, pp. 849-852, 1989.

[20] S. 1. Braginskii, "Transport processing in plasmas,"' in Review of Modern Physics, vol. 1, New York: Consultants Bureau, 1965.

[21] C. Keane and C. H. Skinner, "Radiative power electron cooling rates for oxygen in steady-state and transient plasmas at densities beyond the corona limit," Phys. Rev. A, vol. 33, pp. 4179-4190, 1986.

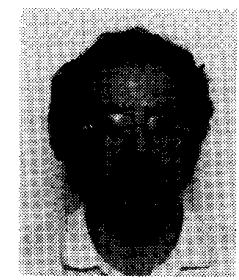

Mario C. Marconi was born in Buenos Aires, Argentina, on March 7, 1955. He received the degrees of Licenciado en Fisica and Doctor en Fisica from the Universidad de Buenos Aires in 1980 and 1985 , respectively.

He joined CEILAP (CITEFA) in 1978, where he worked in new techniques to obtain short pulse emission in solid-state lasers. Since 1986 he has been with Colorado State University; Fort Collins, under a Postdoctoral Fellowship from the Universidad de Buenos Aires and the Consejo Nacional de Investigaciones Cientificas y Tecnicas (CONICET).

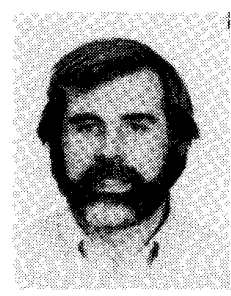

Jorge J. Rocea (S'80-M'83) received the diploma in physics from the University of Rosario, Argentina in 1978 and the Ph.D. degree from Colorado State University in 1983.

He is currently an Associate Professor with the Department of Electrical Engineering, Colorado State University. His broad research interests include the development of new short-wavelength lasers and the physics of discharges and plasmas His work has included the development of ion lasers excited by electron beams, the generation of 
intense electron beams from glow discharges, the demonstration of recombination lasing in negative glow plasmas and the study of dense capillary discharges which he has proposed as possible source of $\mathrm{X}$-ray and XUV recombination laser radiation.

Dr. Rocca received a National Science Foundation Presidential Young Investigator Award for 1985-1990 and he is also a recipient of the Halliburton Foundation Award. He is a member of the Optical Society of America.

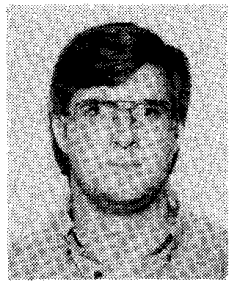

John F. Schmerge was born in Storm Lake, IO on November 30,1965 . He received the B.S. degree in electrical engineering from Colorado State University, Fort Collins, in 1988

He is currently working towards the M.S. degree in electrical engineering at Colorado State University while supported by a National Science Foundation Graduate Fellowship. His research is in the area of gaseous and semiconductor plasmas.

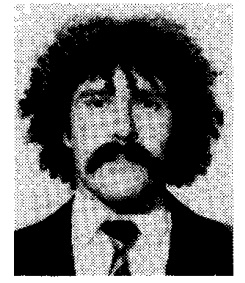

Mayo Villagran was born in Montevideo, Uruguay on May 2, 1954. He received the diploma in physics and the doctor in physics degrees from the University of La Plata, Argentina in 1978 and 1986, respectively.

From 1986 to 1988 he was with Colorado State University with a Fellowship from the Organization of America States. He is presently working at the Universidad Autonoma de Mexico, developing medical applications of lasers.

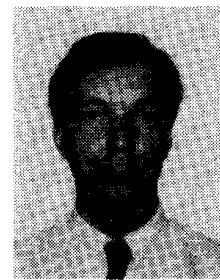

Frank J. Lehmann received the B.S. degree from the University of Washington, Seattle, the M.S degree from Yale University, New Haven, CT, and the Ph.D. degree from Colorado State University, Fort Collins, all in physics in 1981, 1983 and 1986, respectively. In 1987 he was a post doctoral candidate at Colorado State University working with Dr. J. J. Rocca.

Since 1987, he has been with the Lockheed Advanced Development Company, Burbank, CA His research interests include optical remote sensing, diagnostics, and laser design.

Dr. Lehmann is a member of the American Physical Society, the Optical Society of America, and Phi Beta Kappa. 